\title{
Carmen Heine 2010. Modell zur Produktion von Online-Hilfen. Berlin: Frank \& Tim- me. 315 Seiten, 44,80 Euro. ISBN 978-3-86596-263-8
}

Software-Hilfetexte stellen den Textproduzenten vor vielfache Anforderungen: Unterschiedlich versierte Anwender müssen durch die Software geführt werden; mögliche Bedienungsschwierigkeiten oder Aufgaben, die mit der Software ausgeführt werden sollen, müssen bei der Produktion des Hilfetextes bereits antizipiert werden; die Software muss beschrieben werden können, hierfür ist es notwendig, dass der Hilfetext-Produzent mit dem Produkt arbeiten kann. Ist der Produktionsprozess der Online-Hilfe von der Entwicklung des Produkts selbst abgekoppelt, führt dies zu ungeahnten Schwierigkeiten. An dieser Stelle setzt Carmen Heine mit ihrer Dissertation „Modell zur Produktion von Online-Hilfen“ an, die im Frank \& Timme-Verlag erschienen ist. Sie verbindet die Anforderungen der Textproduktion mit den Gewohnheiten der Softwareentwicklung und entwickelt daraus ihr Modell.

Zunächst erläutert sie die Textsorte „Online-Hilfe“, wobei sie elegant ihr Wissen um verschiedene Leserkreise einsetzt. Sie erklärt Fachbegriffe aus der technischen Dokumentation in Fußnoten und kann sich auf diese Art und Weise im Haupttext auf die Darstellung von Inhalten konzentrieren. Zentral für die Produktion von Online-Hilfen ist das Hypertext-Konzept. Heine führt hierzu verschiedene Konzeptionen und Begriffe ein und setzt sie mit ihrer Fragestellung in Beziehung. In ihrer textsortensystematischen Betrachtung konstatiert sie ein Forschungsdesiderat, da die Textsorte nur teilweise konventionalisiert ist (S. 59) und daher nicht für alle Anforderungen standardisiertes Textmusterwissen vorliegt (S. 60).

Im Kapitel „Produktionsprozess und Ontogenese“ verknüpft sie den Prozess der Softwareentwicklung mit dem der Softwaredokumentation und setzt sich kritisch mit vorhandenen Ansätzen auseinander, indem sie Bereiche aufzeigt, die von den vorgestellten Modellen nicht oder unzureichend präzise ausformuliert sind. Die in diesem Kapitel implizit formulierten Forderungen an ein Modell greift sie in ihrer eigenen Modellierung wieder auf und setzt sie dort um.

Ein Überblick über Ansätze der Schreibprozessforschung und Modellierungen des Schreibprozesses wird im vierten Kapitel gegeben. Heine stellt jedoch nicht nur die verschiedenen, in der Schreibforschung etablierten Ansätze vor, sondern versteht es, neuere Ansätze gezielt auf ihre Fragestellung zu beziehen. So stellt sie das Modell für die Ontogenese des Schreibprozesses von Feilke/Augst (1989) der Spezifikation der Wissensbereiche von Rothkegel (2005) gegenüber, vergleicht sie, zieht Konsequenzen für ihre eigene Modellierung und zeichnet somit die Herkunft ihrer Gedanken nach. Darüber hinaus bezieht sie fachpraktische Modellierungen mit ein, da diese den besonderen Anforderungen der technischen Redaktion Rechnung tragen. Heine schließt dieses Kapitel mit einer tabellarischen Zusammenfassung, in der sie die in den Modellen betrachteten Aspekte, die für die Produktion von Online-Hilfe relevant sind, aufführt und beschreibt, wie sie diese in ihr Modell integrieren wird und ermöglicht dem Leser dadurch einen übersichtlichen Vergleich.

Heines Dissertation ist - an dieser Stelle sei diese Bemerkung im Angesicht des aktuellen Diskurses um die Korrektheit von Bezugnahmen erlaubt - ein hervorragendes Beispiel für den korrekten Umgang mit dem Gedankengut Anderer.

Das Kernstück ist das „Online-Hilfe-Produktionsmodell“, das in Kapitel 5 vorgestellt wird. Sehr selbstbewusst geht Heine hier mit notwendigen Einschränkungen ihrer Modellierung um, indem sie sich der „Methode der Verallgemeinerung“ bedient und ihr Modell „im Bereich der Wissenskomponenten ... besonders abstrakt angelegt“ bezeichnet und den „Entwurfscharakter“ betont, worin sie jedoch im Hinblick auf „Prüfbarkeit und Offenheit für Anschlusshypothesen“ des Modells keinen Nachteil erkennt (S. 173). Ein weiteres Indiz dafür, dass Heine sich sehr sicher in ihrem Gegenstandsbereich fühlt, ist ihr Verzicht auf einen Beleg oder Rückverweis für die „direkte Übernahme“ von Teilen des Online-Hilfe-Produktionsmodells aufgrund der Tatsache, dass „sie auf gesicherten Erkenntnissen der Schreib- und Fachkommunikationsforschung und des Projektmanagement beruhen“ (S. 176). Allerdings könnte ein zweifelnder Geist diese Behauptungen in 
Frage stellen.

Inhaltlich zeigt sich die Komplexität und Anschaulichkeit der Modellierung auf den weiteren Seiten. Ihr Produktionsmodell gliedert Heine in drei Bereiche. In „Bereich 1“ fokussiert sie den Produktionsprozess, der mit der Auftragserteilung beginnt und durch verschiedene Phasen bis hin zum Prototyping bzw. zum Recycling modelliert ist. Die Produktionsphasen verbindet sie über die Kognition mit den Ontogenesephasen, wobei sie die „Kognition“ in Anlehnung an das didaktikorientierte Schreibprozessmodell von Göpferich (2002) spezifiziert. Die Leistung von Heine besteht also darin, die verschiedenen Ansätze nutzbringend für die Modellierung einer spezifischen Produktionsart, nämlich die der Online-Hilfe-Produktion, zu kombinieren.

Im „Bereich 2“ differenziert Heine Wissenstypen und schlägt dabei einen neuen Weg ein: Sie fasst Wissen, das in anderen Kompetenzmodellen als prozedurales und deklaratives Wissen unterschieden wird (so z. B. in Becker-Mrotzek/Schindler 2007), als „Sachwissen“ zusammen, da diese zusammengenommen die Voraussetzung für das Gelingen der Produktion einer Online-Hilfe bilde (S. 204). Darüber hinaus bestimmt Heine noch Text-, Dokumentations- und Medienwissen als relevante und individuell geprägte Wissensbereiche, die den Produktionsprozess beeinflussen. Die empirische Überprüfung ihres Modells rechtfertigt und belegt die Differenzierung dieser Wissensbereiche. Sie zeigt u. a., dass Experten aufgrund ihres Medien- und Dokumentationswissens andere Strategien beim Produzieren von Online-Hilfen entwickeln (können) als Laien.

Die situativen Bedingungen betrachtet Heine als „Bereich 3“. Die Berücksichtigung einer erweiterten Textproduktionssituation ist seit langem in der Schreibprozessforschung üblich und daher nicht ganz so neu, wie Heine es darstellt. Das gleiche gilt für das wechselseitige Zusammenspiel der beteiligten Komponenten.

Insgesamt stellt Heine mit ihrem Modell einen Ansatz vor, der durch seine präzise Beschreibung sehr gut nachvollzieh- und anwendbar ist. Sie unterzieht ihr Modell einer empirischen Überprüfung. Hierfür konstruiert sie eine Aufgabe, in der Versuchspersonen ein Online-Hilfe-Text erstellen sollen. Beeindruckend ist hierbei die methodische Herangehensweise: Es werden nicht nur Logdaten der Eingaben erfasst, sondern gleichzeitig Protokolle Lauten Denkens erstellt. In acht Fällen wurden zudem die Augenbewegungen aufgezeichnet, um zwischen den Daten noch besser triangulieren zu können. Allerdings führten technische Schwierigkeiten dazu, dass die Eyetracking Daten von Heine nur ergänzend genutzt werden konnten. Über einen Fragebogen wurden Sprachkenntnisse, berufliche Qualifikationen sowie Einschätzungen zu eigenen Fertigkeiten erhoben.

Die qualitative Datenauswertung führt Heine mit dem Ziel durch, die einzelnen Komponenten ihres Modells durch Äußerungen oder Handlungen zu bestätigen, indem sie hierfür Belege aus den Daten anführt und erläutert. Dass sie für jede Modellkomponente Entsprechungen in den Daten findet, interpretiert sie als Bestätigung ihres Modells. Selbstkritisch hinterfragt sie ihre Unterscheidung zwischen „Semi-Professionellen“ und „Experten“ (S. 288), die sich in der Herangehensweise an die Aufgabe zeigen und keine signifikanten Ergebnisse zur Modellevaluation beitragen. Der ontogenetische Zugang und die Einbeziehung der verschiedenen Wissensbereiche erweist sich als nützlich für diese spezifische Form der Textproduktion und zeigt somit einen Weg der Weiterentwicklung von Modellierungen auf.

Das umfangreiche Datenmaterial ist dem Buch auf einer CD beigefügt, so dass man die Aussagen Heines an den Transkripten nachvollziehen kann, wenn man es möchte. An der einen oder anderen Stelle wären weitere Querverweise auf das Datenmaterial hilfreich gewesen, um dem Eindruck entgegenzuwirken, es handele sich bei den Äußerungen um Behauptungen.

Das Lesevergnügen wird ab und zu dadurch getrübt, dass eingebundene Abbildungen so klein sind, dass sie nur schwer (S. 55, 56) oder gar nicht (S. 220) mehr lesbar sind. Dies ist umso bedauerlicher, wenn die Abbildung nicht nur illustrierenden Charakter haben, sondern wie auf S. 220 verhindern, dass die Argumentation, die auf der Gegenüberstellung von Aufgabenstellung und Aufgabenbearbeitung beruht, nicht nachvollzogen werden kann. 
An einigen Stellen sind noch Spuren des Schreibprozesses sichtbar, die eigentlich in einer Endredaktion hätten getilgt werden müssen. Hier zeigt sich der Nachteil der Veränderung der Publikationskultur, in der zunehmend auf Lektorate verzichtet wird.

Insgesamt hat Carmen Heine mit ihrem Buch „Modell zur Produktion von Online-Hilfen“ eine beeindruckende Dissertation veröffentlicht, die für Leser geeignet ist, die Genauigkeit in der Darstellung von Übernahmen fremden Gedankenguts verlangen und die einen Fachtext leserfreundlich aufbereitet rezipieren, sich über die Ansätze der Schreibforschung und ihre Weiterentwicklung informieren sowie einen innovativen Ansatz kennenlernen möchten. Ihre Dissertation trägt zur Verknüpfung von technischer Dokumentation und Schreibforschung bei und ist für die Praxis ebenso wie für die Forschung ein Gewinn.

Dagmar Knorr

\section{Literatur}

Becker-Mrotzek, Michael/Schindler, Kirsten (2007): Schreibkompetenzen modellieren. In Becker-Mrotzek, Michael/ Schindler, Kirsten (Hrsg.), Texte schreiben. Duisburg: Gilles \& Francke [KöBeS - Kölner Beiträge zur Sprachdidaktik/Reihe A, 5], 7-26

Feilke, Helmuth/Augst, Gerhard (1989): Zur Ontogenese der Schreibkompetenz. In Antos, Gerd/Krings, Hans P. (Hrsg.), Textproduktion. Ein interdisziplinärer Forschungsüberblick. Tübingen: Niemeyer [Konzepte der Sprach- und Literaturwissenschaft; 48], 297-327

Göpferich, Susanne (2002): Textproduktion im Zeitalter der Globalisierung. Entwicklung einer Didaktik des Wissenstransfers. Tübingen: Stauffenburg

Rothkegel, Annely (2005): Zur Modellierung von Schreibaufgaben. In Jakobs, Eva-Maria/Lehnen, Katrin/Schindler, Kirsten (Hrsg.), Schreiben am Arbeitsplatz. Wiesbaden: VS Verlag für Sozialwissenschaften [Schreiben - Medien - Beruf], 57-72. 